\title{
On Almost Generalized Weakly Symmetric $\alpha$-Cosymplectic Manifolds
}

\author{
Mustafa Yıldırım ${ }^{*}$ and Selahattin Beyendi ${ }^{2}$ \\ ${ }^{1}$ Aksaray University, Faculty of Art and Scinence, Department of Mathematics, Aksaray, Turkey \\ ${ }^{2}$ Inönü University, Faculty of Education, 44000, Malatya, Turkey \\ * Corresponding author
}

\section{Article Info}

Keywords: Almost generalized weakly symmetric manifold, Almost generalized weakly Ricci-symmetric manifold, $\alpha$-cosymplectic manifold.

2010 AMS: 53C15,53C25.

Received: 2 May 2020

Accepted: 29 September 2020

Available online: 23 December 2020

\begin{abstract}
In the present paper, we study the notions of an almost generalized weakly symmetric $\alpha$ cosymplectic manifolds and an almost generalized weakly Ricci-symmetrik $\alpha$-cosymplectic manifolds.
\end{abstract}

\section{Introduction}

In 1989, L. Tamassy and T. Q. Binh intruduced the notions of weakly symmetric Riemannian manifold [10]. In the view of [5], a non flat $(2 n+1)$-dimensional differantiable manifold, $n>1$, is called almost weakly pseudo symmetric manifold, if there exist $A_{1}, B_{1}, C_{1}, D_{1},($ are non-zero) 1-forms on $M$ such that

$$
\begin{aligned}
\left(\nabla_{W} R\right)\left(X_{1}, X_{2}, X_{3}, X_{4}\right) & =\left[A_{1}(W)+B_{1}(W)\right] R\left(X_{1}, X_{2}, X_{3}, X_{4}\right)+C_{1}\left(X_{1}\right) R\left(W, X_{2}, X_{3}, X_{4}\right) \\
& +C_{1}\left(X_{2}\right) R\left(X_{1}, W, X_{3}, X_{4}\right)+D_{1}\left(X_{3}\right) R\left(X_{1}, X_{2}, W, X_{4}\right)+D_{1}\left(X_{4}\right) R\left(X_{1}, X_{2}, X_{3}, W\right),
\end{aligned}
$$

where $R\left(X_{1}, X_{2}, X_{3}, X_{4}\right)=g\left(R\left(X_{1}, X_{2}\right) X_{3}, X_{4}\right), R$ is curvature tensor of type $(1,3), A_{1}, B_{1}, C_{1}, D_{1}$ are non-zero 1 -forms defined by $A_{1}(W)=$ $g\left(W, \sigma_{1}\right), B_{1}(W)=g\left(W, \rho_{1}\right), C_{1}(W)=g\left(W, \pi_{1}\right), D_{1}(W)=g\left(W, \partial_{1}\right)$ and $\sigma_{1}, \rho_{1}, \pi_{1}, \partial_{1}$ are vector fields metrically equivalent to the 1 -forms, for all $W$. Also $\nabla$ denotes Levi-Civita connection with respect to metric tensor $g$. A $(2 n+1)$-dimensional Riemannian manifold of this kind is denoted by $(W S)_{2 n+1}$-manifold.

Dubey [8] presented generalized recurrent space. In keeping with this work, we shall call a $(2 n+1)$-dimensional $\alpha$-cosymplectic manifold almost generalized weakly symmetric ( briefly $(G W S)_{2 n+1}$-manifold) if admits the equation

$$
\begin{aligned}
\left(\nabla_{W} R\right)\left(X_{1}, X_{2}, X_{3}, X_{4}\right) & =\left[A_{1}(W)+B_{1}(W)\right] R\left(X_{1}, X_{2}, X_{3}, X_{4}\right)+C_{1}\left(X_{1}\right) R\left(W, X_{2}, X_{3}, X_{4}\right) \\
& +C_{1}\left(X_{2}\right) R\left(X_{1}, W, X_{3}, X_{4}\right)+D_{1}\left(X_{3}\right) R\left(X_{1}, X_{2}, W, X_{4}\right) \\
& +D_{1}\left(X_{4}\right) R\left(X_{1}, X_{2}, X_{3}, W\right)+\left[A_{2}(W)+B_{2}(W)\right] G\left(X_{1}, X_{2}, X_{3}, X_{4}\right) \\
& +C_{2}\left(X_{1}\right) G\left(W, X_{2}, X_{3}, X_{4}\right)+C_{2}\left(X_{2}\right) G\left(X_{1}, W, X_{3}, X_{4}\right) \\
& +D_{2}\left(X_{3}\right) G\left(X_{1}, X_{2}, W, X_{4}\right)+D_{2}\left(X_{4}\right) G\left(X_{1}, X_{2}, X_{3}, W\right)
\end{aligned}
$$

where

$$
G\left(X_{1}, X_{2}, X_{3}, X_{4}\right)=\left[g\left(X_{2}, X_{3}\right) g\left(X_{1}, X_{4}\right)-g\left(X_{1}, X_{3}\right) g\left(X_{2}, X_{4}\right)\right]
$$

and $A_{i}, B_{i}, C_{i}, D_{i},(i=1,2)$, are non-zero 1 -forms defined by $A_{i}(W)=g\left(W, \sigma_{i}\right), B_{i}(W)=g\left(W, \rho_{i}\right), C_{i}(W)=g\left(W, \pi_{i}\right)$ and $D_{i}(W)=g\left(W, \partial_{i}\right)$. There are interesting results of such $(G W S)_{2 n+1}$-manifold is that it has kind of 
i) (for $A_{i}=B_{i}=C_{i}=D_{i}=0$ ), locally symmetric space in the sense of Cartan

ii) (for $A_{1} \neq 0, B_{i}=C_{i}=D_{i}=0$ ), recurrent space by Walker [13],

iii) (for $A_{i} \neq 0, B_{i}=C_{i}=D_{i}=0$ ), generalized reccurent space by Dubey [8],

iv) (for $A_{1}=B_{1}=C_{1}=D_{1} \neq 0$ and $A_{2}=B_{2}=C_{2}=D_{2}=0$ ), pseudo symmetric space by Chaki [6],

v) (for $A_{1}=-B_{1}, C_{1}=D_{1}$ and $A_{2}=B_{2}=C_{2}=D_{2}=0$ ), semi-pseudo symmetric space in the sense of Tarafdar et al. [11],

vi) ( for $A_{1}=-B_{1}, C_{1}=D_{1}$ and $A_{2}=-B_{2}, C_{2}=D_{2}=0$ ), generalized semi-pseudo symmetric space in the sense of Baishya [3],

vii) (for $A_{i}=B_{i}=C_{i}=D_{i} \neq 0$ ), generalized pseudo symmetric space, by Baishya [3]

viii) (for $B_{1} \neq 0, A_{1}=C_{1}=D_{1} \neq 0$ and $A_{2}=B_{2}=C_{2}=D_{2}=0$ ), almost pseudo symmetric space in the sprite of Chaki et al [5],

ix) (for $B_{i} \neq 0, A_{i}=C_{i}=D_{i} \neq 0$ ), almost generalized pseudo symmetric space in the sense of Baishya,

x) (for $A_{2}=B_{2}=C_{2}=D_{2}=0$ ), weakly symmetric space by Tamassy and Binh [10].

Recently, $\alpha$-cosymplectic manifolds and almost $\alpha$-cosymplectic manifolds have been studied by many different researchers ( [1], [2] [4], [9]). Motivated by the above studies, we consider an almost generalized weakly symmetric $\alpha$-cosymplectic manifolds and an almost generalized weakly Ricci-symmetric $\alpha$-cosymplectic manifold also obtain some interesting results.

\section{Preliminaries}

Let $M^{2 n+1}$ be a connected almost contact metric manifold with an almost contact metric structure $(\varphi, \xi, \eta, g)$, that is, $\varphi$ is a tensor field, $\xi$ is a vector field, $\eta$ is a 1 -form and $g$ is a compatible Riemannian metric such that

$$
\varphi \xi=0, \quad \eta(\varphi W)=0, \quad \eta(\xi)=1
$$

$$
\begin{aligned}
& \varphi^{2} W=-W+\eta(W) \xi, \quad g(W, \xi)=\eta(W), \\
& g\left(\varphi W, \varphi X_{1}\right)=g\left(W, X_{1}\right)-\eta(W) \eta\left(X_{1}\right),
\end{aligned}
$$

for any vector fields $W$ and $X_{1}$ on $M^{2 n+1}$ [7].

If moreover

$$
\begin{aligned}
& \nabla_{W} \xi=-\alpha \varphi^{2} W, \\
& \left(\nabla_{W} \eta\right) X_{1}=\alpha\left[g\left(W, X_{1}\right)-\eta(W) \eta\left(X_{1}\right)\right],
\end{aligned}
$$

where $\nabla$ denotes the Riemannian connection of hold and $\alpha$ is a real number, then $\left(M^{2 n+1}, \varphi, \xi, \eta, g\right)$ is called an $\alpha$-cosymplectic manifold [12]. In this case, it is well know that [9]

$$
\begin{aligned}
& R\left(W, X_{1}\right) \xi=\alpha^{2}\left[\eta(W) X_{1}-\eta\left(X_{1}\right) W\right], \\
& S(W, \xi)=-2 n \alpha^{2} \eta(W), \\
& S(\xi, \xi)=-2 n \alpha^{2},
\end{aligned}
$$

where $S$ denotes the Ricci tensor. From (2.4), it easily follows that

$$
\begin{aligned}
& R(W, \xi) X_{1}=\alpha^{2}\left[g\left(W, X_{1}\right) \xi-\eta\left(X_{1}\right) W\right] \\
& R(W, \xi) \xi=\alpha^{2}[\eta(W) \xi-W],
\end{aligned}
$$

for any vector fields $W, X_{1}, Z$ where $R$ is the Riemannian curvature tensor of the manifold. An $\alpha$-cosymplectic manifold is said to be an $\eta$-Einstein manifold if Ricci tensor $S$ satisfies condition

$$
S\left(W, X_{1}\right)=\lambda_{1} g\left(W, X_{1}\right)+\lambda_{2} \eta(W) \eta\left(X_{1}\right),
$$

where $\lambda_{1}, \lambda_{2}$ are certain scalars.

\section{Almost generalized weakly symmetric $\alpha$-cosymplectic manifold}

An $\alpha$-cosymplectic manifold $\left(M^{2 n+1}, g\right)$ is said to be an almost generalized weakly symmetric if admits the relation (1.1), $(n \geq 1)$. Now, contracting $X_{1}$ over $X_{4}$ in both sides of (1.1), we obtain

$$
\begin{aligned}
\left(\nabla_{W} S\right)\left(X_{2}, X_{3}\right) & =\left[A_{1}(W)+B_{1}(W)\right] S\left(X_{2}, X_{3}\right)+C_{1}\left(R\left(W, X_{2}\right) X_{3}\right)+C_{1}\left(X_{2}\right) S\left(W, X_{3}\right)+D_{1}\left(X_{3}\right) S\left(W, X_{2}\right)+D_{1}\left(R\left(W, X_{3}\right) X_{2}\right) \\
& +2 n\left[A_{2}(W)+B_{2}(W)\right] g\left(X_{2}, X_{3}\right)+C_{2}\left(G\left(W, X_{2}\right) X_{3}\right)+2 n C_{2}\left(X_{2}\right) g\left(W, X_{3}\right)+2 n D_{2}\left(X_{3}\right) g\left(X_{2}, W\right)+D_{2}\left(G\left(W, X_{3}\right) X_{2}\right) .
\end{aligned}
$$

Putting $X_{3}=\xi$ in (3.1) and using (1.2), (2.4), (2.5), (2.7), we have

$$
\begin{aligned}
\left(\nabla_{W} S\right)\left(X_{2}, \xi\right) & =\left(-2 n \alpha^{2}\right)\left[A_{1}(W)+B_{1}(W)\right] \eta\left(X_{2}\right)+(-2 n+1) \alpha^{2} C_{1}\left(X_{2}\right) \eta(W) \\
& -\alpha^{2} C_{1}(W) \eta\left(X_{2}\right)+D_{1}(\xi) S\left(X_{2}, W\right)+\alpha^{2} g\left(W, X_{2}\right) D_{1}(\xi)-\alpha^{2} D_{1}(W) \eta\left(X_{2}\right) \\
& +2 n\left[A_{2}(W)+B_{2}(W)\right] \eta\left(X_{2}\right)+C_{2}(W) \eta\left(X_{2}\right)-C_{2}\left(X_{2}\right) \eta(W) \\
& +2 n C_{2}\left(X_{2}\right) \eta(W)+2 n D_{2}(\xi) g\left(W, X_{2}\right)+D_{2}(W) \eta\left(X_{2}\right)-D_{2}(\xi) g\left(W, X_{2}\right) .
\end{aligned}
$$


Taking $X_{3}=\xi$ in the below identity

$\left(\nabla_{W} S\right)\left(X_{2}, X_{3}\right)=\nabla_{W} S\left(X_{2}, X_{3}\right)-S\left(\nabla_{W} X_{2}, X_{3}\right)-S\left(X_{2}, \nabla_{W} X_{3}\right)$

and then using (2.2), (2.3), (2.5), we obtain

$$
\left(\nabla_{W} S\right)\left(X_{2}, \xi\right)=2 n \alpha^{2} g\left(X_{2}, W\right)-\alpha^{2} S\left(X_{2}, W\right) .
$$

Now, using (3.3) in (3.2), we have

$$
\begin{aligned}
2 n \alpha^{2} g\left(X_{2}, W\right)-\alpha^{2} S\left(X_{2}, W\right) & =-2 n \alpha^{2}\left[A_{1}(W)+B_{1}(W)\right] \eta\left(X_{2}\right)+(-2 n+1) \alpha^{2} C_{1}\left(X_{2}\right) \eta(W) \\
& -\alpha^{2} C_{1}(W) \eta\left(X_{2}\right)+D_{1}(\xi) S\left(X_{2}, W\right)+\alpha^{2} g\left(W, X_{2}\right) D_{1}(\xi) \\
& -\alpha^{2} D_{1}(W) \eta\left(X_{2}\right)+2 n\left[A_{2}(W)+B_{2}(W)\right] \eta\left(X_{2}\right)+C_{2}(W) \eta\left(X_{2}\right) \\
& -C_{2}\left(X_{2}\right) \eta(W)+2 n C_{2}\left(X_{2}\right) \eta(W)+2 n D_{2}(\xi) g\left(W, X_{2}\right) \\
& +D_{2}(W) \eta\left(X_{2}\right)-D_{2}(\xi) g\left(W, X_{2}\right) .
\end{aligned}
$$

Then replacing $W$ and $X_{2}$ by $\xi$ in (3.4) and (2.1), (2.6), we get

$$
\alpha^{2}\left[A_{1}(\xi)+B_{1}(\xi)+C_{1}(\xi)+D_{1}(\xi)\right]=A_{2}(\xi)+B_{2}(\xi)+C_{2}(\xi)+D_{2}(\xi) .
$$

In particular, if $A_{2}(\xi)=B_{2}(\xi)=C_{2}(\xi)=D_{2}(\xi)=0$, formula (3.5) turns into

$$
\alpha^{2}\left[A_{1}(\xi)+B_{1}(\xi)+C_{1}(\xi)+D_{1}(\xi)\right]=0 .
$$

Theorem 3.1. In an almost generalized weakly symmetric $\alpha$-cosymplectic manifold $\left(M^{2 n+1}, g\right), n \geq 1$, the relation (3.5) hold good. Again from (3.1), putting $X_{2}=\xi$, we have

$$
\begin{aligned}
-2 n \alpha^{3} g\left(X_{3}, W\right)-\alpha S\left(X_{3}, W\right) & =\left[A_{1}(W)+B_{1}(W)\right] S\left(\xi, X_{3}\right)+C_{1}\left(R(W, \xi) X_{3}\right)+C_{1}(\xi) S\left(W, X_{3}\right) \\
& +D_{1}\left(X_{3}\right) S(W, \xi)+D_{1}\left(R\left(W, X_{3}\right) \xi\right)+2 n\left[A_{2}(W)+B_{2}(W)\right] g\left(\xi, X_{3}\right) \\
& +C_{2}(W) g\left(\xi, X_{3}\right)-C_{2}(\xi) g\left(W, X_{3}\right)+2 n C_{2}(\xi) g\left(W, X_{3}\right) \\
& +2 n D_{2}\left(X_{3}\right) g(\xi, W)+D_{2}(W) g\left(\xi, X_{3}\right)-D_{2}\left(X_{3}\right) g(\xi, W) .
\end{aligned}
$$

Using (2.4), (2.5), (2.7) in (3.6), we obtain

$$
\begin{aligned}
-2 n \alpha^{3} g\left(X_{3}, W\right)-\alpha S\left(X_{3}, W\right) & =-2 n \alpha^{2}\left[A_{1}(W)+B_{1}(W)\right] \eta\left(X_{3}\right)+\alpha^{2} C_{1}(\xi) g\left(W, X_{3}\right)-\alpha^{2} \eta\left(X_{3}\right) C_{1}(W) \\
& +C_{1}(\xi) S\left(W, X_{3}\right)-2 n \alpha^{2} \eta(W) D_{1}\left(X_{3}\right)+\alpha^{2} \eta(W) D_{1}\left(X_{3}\right) \\
& -\alpha^{2} \eta\left(X_{3}\right) D_{1}(W)+2 n\left[A_{2}(W)+B_{2}(W)\right] \eta\left(X_{3}\right)+C_{2}(W) \eta\left(X_{3}\right) \\
& +2 n D_{2}\left(X_{3}\right) \eta(W)+D_{2}(W) \eta\left(X_{3}\right)-D_{2}\left(X_{3}\right) \eta(W) .
\end{aligned}
$$

Putting $X_{3}=\xi$ in (3.7), we get

$$
\begin{aligned}
\alpha^{2}\left[2 n\left(A_{1}(W)+B_{1}(W)\right)+C_{1}(W)+D_{1}(W)\right]+(2 n-1) \alpha^{2}\left[C_{1}(\xi)+D_{1}(\xi)\right] \eta(W) & =2 n\left[A_{2}(W)+B_{2}(W)\right]+C_{2}(W)+D_{2}(W) \\
& +(2 n-1)\left[C_{2}(\xi)+D_{2}(\xi)\right] \eta(W) .
\end{aligned}
$$

Using $W=\xi$ in (3.7), we obtain

$$
\begin{aligned}
& 2 n \alpha^{2}\left[A_{1}(\xi)+B_{1} \xi+C_{1}(\xi)\right] \eta\left(X_{3}\right)+\alpha^{2} D_{1}(\xi) \eta\left(X_{3}\right)+(2 n-1) \alpha^{2} D_{1}\left(X_{3}\right) \\
& =2 n\left[A_{2}(\xi)+B_{2}(\xi)+C_{2}(\xi)\right] \eta\left(X_{3}\right)+D_{2}(\xi) \eta\left(X_{3}\right)+(2 n-1) D_{2}\left(X_{3}\right) .
\end{aligned}
$$

Replacing $X_{3}$ by $W$ in the above equation and using (3.5), we have

$$
\alpha^{2} D_{1}(\xi) \eta(W)-\alpha^{2} D_{1}(W)=D_{2}(\xi) \eta(W)-D_{2}(W) .
$$

Again, putting $W=\xi$ in (3.4), we get

$$
\begin{aligned}
& 2 n \alpha^{2}\left[A_{1}(\xi)+B_{1}(\xi)+D_{1}(\xi)\right] \eta\left(X_{2}\right)+\alpha^{2} C_{1}(\xi) \eta\left(X_{2}\right)+(2 n-1) \alpha^{2} C_{1}\left(X_{2}\right) \\
& =2 n\left[A_{2}(\xi)+B_{2}(\xi)+D_{2}(\xi)\right] \eta\left(X_{2}\right)+C_{2}(\xi) \eta\left(X_{2}\right)+(2 n-1) C_{2}\left(X_{2}\right) .
\end{aligned}
$$

Replacing $X_{2}$ by $W$ in (3.10) and using (3.5), we obtain

$$
\alpha^{2} C_{1}(\xi) \eta(W)-\alpha^{2} C_{1}(W)=C_{2}(\xi) \eta(W)-C_{2}(W) .
$$

Subtracting (3.9), (3.11) from (3.8)

$$
\alpha^{2}\left[A_{1}(W)+B_{1}(W)+C_{1}(W)+D_{1}(W)\right]=\left[A_{2}(W)+B_{2}(W)+C_{2}(W)+D_{2}(W)\right] .
$$

Next, in view of $A_{2}=B_{2}=C_{2}=D_{2}=0$, the relation (3.12) yields

$$
\alpha^{2}\left[A_{1}(W)+B_{1}(W)+C_{1}(W)+D_{1}(W)\right]=0 .
$$

This motivates us to state the followings

Theorem 3.2. In an almost generalized weakly symmetric $\alpha$-cosymplectic manifold $\left(M^{2 n+1}, g\right)(n \geq 1)$, the sum of the associated 1-forms is given by (3.12).

Theorem 3.3. There does not exist an $\alpha$-cosymplectic manifold which is

(i) recurrent,

(ii) generalized recurrent provided the 1-forms are collinear,

(iii) pseudo symmetric,

(iv) generalized semi-pseudo symmetric provided the 1-forms are collinear,

(v) generalized almost-pseudo symmetric provided the 1-forms are collinear. 


\section{Almost generalized weakly Ricci-symmetric $\alpha$-cosymplectic manifold}

An $\alpha$-cosymplectic manifold $\left(M^{2 n+1}, g\right)(n \geq 1)$, is said to be almost generalized weakly Ricci-symmetric if there exist 1-forms, $\tilde{A}_{i}, \tilde{B}_{i}, \tilde{C}_{i}$ and $\tilde{D}_{i}$ which satisfy the condition

$$
\begin{aligned}
\left(\nabla_{W} S\right)\left(X_{2}, X_{3}\right) & =\left[\tilde{A}_{1}(W)+\tilde{B}_{1}(W)\right] S\left(X_{2}, X_{3}\right)+\tilde{C}_{1}\left(X_{2}\right) S\left(W, X_{3}\right)+\tilde{D}_{1}\left(X_{3}\right) S\left(X_{2}, W\right)+\left[\tilde{A}_{2}(W)+\tilde{B}_{2}(W)\right] g\left(X_{2}, X_{3}\right) \\
& +\tilde{C}_{2}\left(X_{2}\right) g\left(W, X_{3}\right)+\tilde{D}_{2}\left(X_{3}\right) g\left(X_{2}, W\right) .
\end{aligned}
$$

Putting $X_{3}=\xi$ in (4.1), and using (2.1), (2.5), we get

$$
\begin{aligned}
\left(\nabla_{W} S\right)\left(X_{2}, \xi\right) & =-2 n \alpha^{2}\left[\tilde{A}_{1}(W)+\tilde{B}_{1}(W)\right] \eta\left(X_{2}\right)-2 n \alpha^{2} \tilde{C}_{1}\left(X_{2}\right) \eta(W)+\tilde{D}_{1}(\xi) S\left(X_{2}, W\right)+\left[\tilde{A}_{2}(W)+\tilde{B}_{2}(W)\right] \eta\left(X_{2}\right) \\
& +\tilde{C}_{2}\left(X_{2}\right) \eta(W)+\tilde{D}_{2}(\xi) g\left(X_{2}, W\right) .
\end{aligned}
$$

Using equation (3.3) in (4.2) we get,

$$
\begin{aligned}
-2 n \alpha^{3} g\left(X_{2}, W\right)-\alpha S\left(X_{2}, W\right) & =-2 n \alpha^{2}\left[\tilde{A}_{1}(W)+\tilde{B}_{1}(W)\right] \eta\left(X_{2}\right)-2 n \alpha^{2} \tilde{C}_{1}\left(X_{2}\right) \eta(W)+\tilde{D}_{1}(\xi) S\left(X_{2}, W\right)+\left[\tilde{A}_{2}(W)+\tilde{B}_{2}(W)\right] \eta\left(X_{2}\right) \\
& +\tilde{C}_{2}\left(X_{2}\right) \eta(W)+\tilde{D}_{2}(\xi) g\left(X_{2}, W\right) .
\end{aligned}
$$

Putting $W=X_{2}=\xi$ in (4.3), we have

$$
2 n \alpha^{2}\left[\tilde{A}_{1}(\xi)+\tilde{B}_{1}(\xi)+\tilde{C}_{1}(\xi)+\tilde{D}_{1}(\xi)\right]=\tilde{A}_{2}(\xi)+\tilde{B}_{2}(\xi)+\tilde{C}_{2}(\xi)+\tilde{D}_{2}(\xi) .
$$

Then, taking $W=\xi$ in (4.3), we obtain

$$
2 n \alpha^{2}\left[\tilde{A}_{1}(\xi)+\tilde{B}_{1}(\xi)+\tilde{D}_{1}(\xi)\right] \eta\left(X_{2}\right)+2 n \alpha^{2} \tilde{C}_{1}\left(X_{2}\right)=\left[\tilde{A}_{2}(\xi)+\tilde{B}_{2}(\xi)+\tilde{D}_{2}(\xi)\right] \eta\left(X_{2}\right)+\tilde{C}_{2}\left(X_{2}\right) .
$$

Using $X_{2}=\xi$ in (4.3), we get

$$
2 n \alpha^{2}\left[\tilde{A}_{1}(\xi)+\tilde{B}_{1}(\xi)+\tilde{D}_{1}(\xi)\right] \eta(W)+2 n \alpha^{2} \tilde{C}_{1}(W)=\left[\tilde{A}_{2}(\xi)+\tilde{B}_{2}(\xi)+\tilde{D}_{2}(\xi)\right] \eta(W)+\tilde{C}_{2}(W) .
$$

Replacing $X_{2}$ by $W$ in (4.5) and adding with (4.6), we have

$$
\begin{aligned}
2 n \alpha^{2}\left[\tilde{A}_{1}(W)+\tilde{B}_{1}(W)+\tilde{C}_{1}(W)\right]-\left[\tilde{A}_{2}(W)+\tilde{B}_{2}(W)+\tilde{C}_{2}(W)\right] & =-2 n \alpha^{2}\left[\tilde{A}_{1}(\xi)+\tilde{B}_{1}(\xi)+\tilde{C}_{1}(\xi)+\tilde{D}_{1}(\xi)\right] \eta(W) \\
& +\left[\tilde{A}_{2}(\xi)+\tilde{B}_{2}(\xi)+\tilde{C}_{2}(\xi)+\tilde{D}_{2}(\xi)\right] \eta(W)-2 n \alpha^{2} \tilde{D}_{1}(\xi) \eta(W) \\
& -\tilde{D}_{2}(\xi) \eta(W) .
\end{aligned}
$$

In view of (4.4) the relation (4.7) becomes

$$
2 n \alpha^{2}\left[\tilde{A}_{1}(W)+\tilde{B}_{1}(W) \tilde{C}_{1}(W)\right]+2 n \alpha^{2} \tilde{D}_{1}(\xi) \eta(W)=\left[\tilde{A}_{2}(W)+\tilde{B}_{2}(W)+\tilde{C}_{2}(W)\right]-\tilde{D}_{2}(\xi) \eta(W) .
$$

Then, taking $W=X_{2}=\xi$ in (4.1), we obtain

$$
2 n \alpha^{2}\left[\tilde{A}_{1}(\xi)+\tilde{B}_{1}(\xi)+\tilde{C}_{1}(\xi)\right] \eta\left(X_{3}\right)+2 n \alpha^{2} \tilde{D}_{1}\left(X_{3}\right)=\left[\tilde{A}_{2}(\xi)+\tilde{B}_{2}(\xi)+\tilde{C}_{2}(\xi)\right] \eta\left(X_{3}\right)+\tilde{D}_{2}\left(X_{3}\right) .
$$

In view of (4.4), replacing $X_{3}$ by $W$ in (4.9) and then adding the resultant with (4.8),

$$
\begin{aligned}
2 n \alpha^{2}\left\{\left[\tilde{A}_{1}(W)+\tilde{B}_{1}(W)+\tilde{C}_{1}(W)+\tilde{D}_{1}(W)\right]\right. & \left.+\left[\tilde{A}_{1}(\xi)+\tilde{B}_{1}(\xi)+\tilde{C}_{1}(\xi)+\tilde{D}_{1}(\xi)\right] \eta(W)\right\}=\left[\tilde{A}_{2}(W)+\tilde{B}_{2}(W)+\tilde{C}_{2}(W)+\tilde{D}_{2}(W)\right] \\
& +\left[\tilde{A}_{2}(\xi)+\tilde{B}_{2}(\xi)+\tilde{C}_{2}(\xi)+\tilde{D}_{2}(\xi)\right] \eta(W) .
\end{aligned}
$$

Next, putting (4.4) in (4.10), we get

$$
2 n \alpha^{2}\left[\tilde{A}_{1}(W)+\tilde{B}_{1}(W)+\tilde{C}_{1}(W)+\tilde{D}_{1}(W)\right]=\tilde{A}_{2}(W)+\tilde{B}_{2}(W)+\tilde{C}_{2}(W)+\tilde{D}_{2}(W) .
$$

Theorem 4.1. In an almost generalized weakly Ricci-symmetric $\alpha$-cosymplectic manifold $\left(M^{2 n+1}, g\right), n \geq 1$, the relation (4.11) hold good.

Theorem 4.2. There does not exist an almost generalized weakly Ricci symmetric $\alpha$-cosymplectic manifold which is
i) recurrent,
ii) generalized recurrent provided the 1-forms are collinear,
iii) pseudo symmetric,
iv) generalized semi-pseudo symmetric provided the 1-forms are collinear,
v) generalized almost-pseudo symmetric provided the 1-forms are collinear.

\section{References}

[1] N. Aktan, M. Yıldırım, C. Murathan,Almost f-cosymplectic manifolds, Mediterr. J. Math., 11(2014), $775-787$.

[2] G. Ayar, S.K. Chaubey, M-Projective curvature tensor over cosymplectic manifolds, Differ. Geom. Dyn. Syst., 21(2019), $23-33$.

[3] K.K. Baishya, P.R. Chowdhury, J. Mikes, P. Peska, On almost generalized weakly symmetric Kenmotsu manifolds, Acta Univ. Palacki. Olomuc., Fac. rer. nat., Mathematica, 55(2016), 2, 5-15.

[4] S. Beyendi, G. Ayar, N. Aktan, On a type of $\alpha$-cosymplectic manifolds, Commun. Fac. Sci. Univ. Ank. Ser. A1 Math. Stat., 68(1)(2019), 852-861.

[5] M.C. Chaki, T. Kawaguchi, On almast pseudo Ricci symmetric manifolds, Tensor, 68(1)(2017), 10-14.

[6] M. C. Chaki, On pseudo Ricci symmetric manifolds, , Bulg. J. Physics, 15(1998), 526-531.

[7] D.E. Blair, Contact manifolds in Riemannian geometry, , Lecture Notes in Math. 509, (1976), Springer-Verlag, Berlin.

[8] R.S.D. Dubey, Generalized recurrent spaces, Indian J. Pure Appl. Math., 10(1979), 1508-1513.

[9] H. Öztürk, C. Murathan, N. Aktan, A.T. Vanli, Almost $\alpha$-cosymplectic f-manifolds, (2014), An. Stiint. Univ. Al. I. Cuza Iasi Inform. (N.S.) Matematica, Tomul LX, f.1.

[10] L.Tamassy, T.Q. Binh, On weakly symmetric and weakly projective symmetric Riemannian manifolds, Coll. Math. Soc., J. Bolyai, 56(1989), 663-670.

[11] M. Tarafdar, M.A.A. Jawarneh, Semi-pseudo Ricci symmetric manifold, J. Indian. Inst. of Science., 73(1993), 591-596.

[12] T.W. Kim, H.K. Pak, Canonical foliations of certain classes of almost contact metric structures, , Acta Math, Sinica, Eng. Ser. Aug., 21(4)(2005),

[13] A.G. Walker, On Ruse's space of recurrent curvature, Proc. of London Math. Soc. 52(1950), 36-54. 